\title{
Thermal Boundary Resistance Effect on Non-Equilibrium Energy Transport in Metal-Dielectric Thin Films Heated by Femtosecond Pulse Lasers
}

\author{
Jae Bin Lee and Seong Hyuk Lee* \\ School of Mechanical Engineering, Chung-Ang University, 221 Heuksuk-dong, Dongjak-gu, Seoul 156-756, Korea
}

The aim of this study is to investigate the effect of interfacial thermal boundary resistance (TBR) at a metal-dielectric interface on nonequilibrium energy transport in $\mathrm{Au} / \mathrm{SiO}_{2}$ films heated by femtosecond pulse lasers. In this paper we suggest a combined set of numerical models that include the two-temperature model (TTM) for a metal side and the heat conduction equation for a dielectric layer. In addition, the TBRs between metal and nonmetal layers are calculated using thermal conductance, which is closely associated with the electron-phonon resistance and phonon-phonon resistance. Herein we present the transient and spatial distributions of TBR for a $\mathrm{Au} / \mathrm{SiO} \mathrm{film}$ irradiated by a 100 -fs pulse laser with a $1053 \mathrm{~nm}$ wavelength, which are substantially affected by the phonon temperature and electron-phonon coupling. We also discuss the effect of laser fluence on the TBR and energy transport. The TBR rapidly increases the thermal conductivity at the interface, and becomes dominant at an early stage of laser irradiation over a very short period and then drastically decreases with time. Moreover, the TBR is substantially affected by the electron-phonon coupling and it should be considered for more accurate prediction of the lattice temperature drop at the interface. [doi:10.2320/matertrans.M2011021]

(Received January 13, 2011; Accepted April 7, 2011; Published June 25, 2011)

Keywords: femtosecond pulse laser, metal-dielectric interface, thermal boundary resistance (TBR), electron-phonon coupling factor, non-equilibrium, thin film

\section{Introduction}

In recent years, femtosecond pulse lasers have been regarded as a promising technique in material processing because the thermal affected zone and collateral damage can be substantially reduced due to the time scale difference between the pulse duration and heat diffusion time. Much effort has been made in a wide variety of fields including medical applications, ${ }^{1)}$ material processing, ${ }^{2)}$ molecular spectroscopy, ${ }^{3)}$ ionization and dissociation of polyatomic molecules. ${ }^{4)}$ For such applications of ultra-short pulse lasers, it is necessary to investigate the non-equilibrium process between energy carriers, fast phase transition, theoretical modeling, and the non-linear effect on laser ablation.

Currently, considerable effort has been devoted to the theoretical and experimental study of non-equilibrium energy transport in metallic thin films. Among many theoretical models for femtosecond pulse laser interactions with metals, the two-temperature model (TTM) has been widely used to examine electron-phonon interactions and non-equilibrium energy transport in metallic thin films. ${ }^{5-12)}$ Because the electron-electron interaction time (on the order of a hundred femtoseconds) is shorter than the electron-phonon interaction time (on the order of picoseconds), a strong non-equilibrium occurs between the electrons and phonons under the irradiation of femtosecond laser pulses. Thermal equilibrium among the excited electrons is reached within a few hundred femtoseconds, depending on the excitation energy above the Fermi level. ${ }^{13)}$ Cooling of the electrons occurs because of the electron-phonon energy transport and electronic diffusive motion.

Multilayer thin films have been used to make useful and applicable structures such as superconductors, microelectronic layer packages, ${ }^{14)}$ and multi-layer metal films. ${ }^{15,16)}$

*Corresponding author, E-mail: shlee89@cau.ac.kr
Generally, the existence of thermal boundary resistance (TBR) at the interface between dissimilar films would significantly affect the device performance, and the importance of TBR has been recognized with the development of micro-electromechanical systems (MEMS). ${ }^{14)}$ For these reasons, research reporting the detailed mechanism of the TBR phenomena at solid-solid interfaces has been conducted. ${ }^{17-21)}$ Most researchers have used the diffuse mismatch model (DMM) to describe solid-solid interfacial TBR. This model considers phonon transmission across the interface under the assumption that the electron-phonon coupling resistance can be neglected. ${ }^{18)}$ Majumdar and Reddy ${ }^{20)}$ suggested the TBR model, which accounts for the phononphonon scattering resistance at the interface between metal and nonmetal and the electron-phonon coupling resistance in metals. TBR causes a rapid decrease in the lattice temperature, resulting in thermal damage of solids.

Although abundant research has been performed, the interaction of a femtosecond pulse laser with multilayer metal-dielectric films is poorly understood, and TBR modeling has not been applied to ultra-short pulse laser interaction with multilayer film structures. Moreover, it is impossible to measure the in situ profile of the interfacial resistance due to the very short time scale and because the interfacial layer cannot be determined experimentally. Hence, the effect of TBR on energy transport in metal-dielectric multilayer films irradiated by a femtosecond pulse laser requires further investigation. Thus, the main objective of this study was to numerically investigate the effect of the interfacial TBR in metal-dielectric $\left(\mathrm{Au} / \mathrm{SiO}_{2}\right)$ thin films heated by femtosecond pulse lasers on non-equilibrium energy transport. Prior to the primary simulation, we validated our computational code by comparing the electron temperatures predicted for an $\mathrm{Au}$ single film with previous numerical results and experimental data. We suggest a combined set of energy equations, which include two-temperature equations for a metal side and a 
well-known conduction equation for a dielectric side. The transient evolution of TBR estimated at the interface is also examined in this article, particularly the analysis of the close interaction of TBR with the phonon temperature and electron-phonon coupling.

\section{Numerical Modeling and Computational Details}

\subsection{A combined computational model of metal- dielectric thin films}

According to Majumdar and Reddy, ${ }^{20)}$ the energy transport across metal-dielectric interfaces must occur between electrons and phonons. There are two different energy transport pathways: (1) coupling between electrons of the metal and phonons of the dielectric at the interface; and (2) coupling between electrons and phonons within the metal. We suggest a combination method to numerically simulate a metaldielectric thin film irradiated by ultra-short pulse lasers. The two-temperature model (TTM) has been widely used to simulate the electron-phonon interaction for metals irradiated by an ultra-short pulse laser. Because a focused laser beam diameter is typically much larger than the penetration depth of the metal, one-dimensional analysis is valid, and the spatial and temporal evolutions of the electron and lattice temperatures are expressed as follows: ${ }^{7,12}$

$$
\begin{aligned}
\frac{\partial C_{\mathrm{e}}\left(T_{\mathrm{e}}\right) T_{\mathrm{e}}}{\partial t}= & \frac{\partial}{\partial z}\left(k_{\mathrm{e}}\left(T_{\mathrm{e}}\right) \frac{\partial T_{\mathrm{e}}}{\partial z}\right)-G\left(T_{\mathrm{e}}-T_{\mathrm{l}}\right)+S(z, t) \\
\frac{\partial C_{\mathrm{l}}\left(T_{1}\right) T_{1}}{\partial t}= & G\left(T_{\mathrm{e}}-T_{1}\right) \\
S(z, t)= & \frac{0.939 F(1-R(t))}{t_{\mathrm{p}}} \exp \left(-\int_{0}^{z} \alpha(z, t) d z\right) \\
& \times \exp \left(-2.773 \frac{t^{2}}{t_{\mathrm{p}}{ }^{2}}\right),
\end{aligned}
$$

where $k_{\mathrm{e}}\left(T_{\mathrm{e}}\right)$ is the electron thermal conductivity, $G$ is the electron-phonon coupling factor, and $S(z, t)$ is the laser heating source term. In eq. (3), $R(t), F, t_{\mathrm{p}}$, and $\alpha(z, t)$ represent the reflectivity at the surface, laser fluence, pulse duration, and absorption coefficient, respectively. In TTM, some of the thermal and optical parameters should be carefully modeled to ensure more accurate predictions. First, the electron heat capacity is commonly expressed as a linear function of the electron temperature, $C_{\mathrm{e}}\left(T_{\mathrm{e}}\right)=\gamma T_{\mathrm{e}}$, where $\gamma=\pi^{2} n_{\mathrm{e}} k^{2}{ }_{\mathrm{B}} / 2 \varepsilon_{\mathrm{F}}{ }^{22)}$ Because this Sommerfeld expansion is no longer valid for high electron temperatures, the electron heat capacity is calculated from the following equation: ${ }^{13)}$

$$
C_{\mathrm{e}}\left(T_{\mathrm{e}}\right)=\int_{-\infty}^{\infty} \frac{\partial f\left(\varepsilon, \mu, T_{\mathrm{e}}\right)}{\partial T_{\mathrm{e}}} g(\varepsilon) \varepsilon d \varepsilon,
$$

where $g(\varepsilon)$ is the electron density of states (DOS), $\mu$ is the chemical potential at $T_{\mathrm{e}}$, and $f\left(\varepsilon, \mu, T_{\mathrm{e}}\right)$ is the Fermi distribution function. Based on the Drude theory, ${ }^{23)}$ we calculated the electron thermal conductivity, which can be expressed as

$$
k_{\mathrm{e}}\left(T_{\mathrm{e}}\right)=\frac{1}{3} C_{\mathrm{e}}\left(T_{\mathrm{e}}\right) V_{\mathrm{e}}^{2}\left(T_{\mathrm{e}}\right) \tau_{\mathrm{e}}^{*}\left(T_{\mathrm{e}}\right)
$$

where $V_{\mathrm{e}}$ denotes the electron velocity, and $\tau_{\mathrm{e}}$ is the electronelectron relaxation time with respect to the electron temper- ature. $^{23)}$ It is noted that for $\tau_{\mathrm{e}}$ related to the collision frequency, there are three different regimes depending on the melting temperature and Fermi temperature: the cold solid regime (i), hot plasma regime (ii), and intermediate regime (iii), which correspond to the following expressions: $:^{9,24)}$

$$
\begin{aligned}
& \text { (i) } v=k_{\mathrm{s}}\left(\frac{m_{\mathrm{e}}}{M}\right)^{1 / 2} \frac{J_{\mathrm{i}}}{\hbar} \frac{T_{1}}{T_{\mathrm{D}}}\left(T_{\mathrm{e}}<T_{\text {melt }}\right) \\
& \text { (ii) } v=k_{\mathrm{s}} \frac{Z n_{\mathrm{e}} \ln \Lambda}{T_{\mathrm{e}}^{3 / 2}}\left(T_{\mathrm{e}} \geq T_{\mathrm{F}}\right) \\
& \text { (iii) } v=k_{\mathrm{s}} \frac{V_{\mathrm{e}}}{r_{0}}\left(T_{\text {melt }}<T_{\mathrm{e}}<T_{\mathrm{F}}\right),
\end{aligned}
$$

where the empirical constant $k_{\mathrm{s}}$ can be obtained from experimental data using the interfacial condition. ${ }^{24)}$ Generally in thin film optics, the reflectivity and absorption coefficient can be expressed as $R=\left((n-1)^{2}+k^{2}\right) /((n+$ $1)^{2}+k^{2}$ ) and $\alpha=4 \pi \kappa / \lambda$, respectively, and they are not constant values during laser irradiation. The complex refractive index can be expressed by $n=\left(\left(\varepsilon_{1}+\left(\varepsilon_{1}{ }^{2}+\right.\right.\right.$ $\left.\left.\left.\varepsilon_{2}^{2}\right)^{1 / 2}\right) / 2\right)^{1 / 2}$ and $k=\left(\left(-\varepsilon_{1}+\left(\varepsilon_{1}^{2}+\varepsilon_{2}^{2}\right)^{1 / 2}\right) / 2\right)^{1 / 2}$ where $n$ and $k$ represent the refractive index and the extinction coefficient, respectively. ${ }^{9)}$ The Drude model was used to determine the complex dielectric function, $\varepsilon_{1}+i \varepsilon_{2}$, which is described by $\varepsilon_{1}=1-\left(\omega_{\mathrm{P}}^{2} /\left(\omega_{\mathrm{P}}^{2}+v^{2}\right)\right.$ and $\varepsilon_{2}=v \omega_{\mathrm{P}}^{2} /$ $\left(\omega_{\mathrm{L}}{ }^{3}+v^{2} \omega_{\mathrm{L}}\right){ }^{9,24)}$ Thus, optical characteristics depend highly on the collision frequency, suggesting variation in the reflectivity during laser irradiation.

The electron-phonon coupling factor, $G$, should be carefully chosen in metals because electron-phonon coupling plays a major role in transferring electron energy to the lattice phonon. Previously, Lee et al. ${ }^{25)}$ compared theoretical models for electron-phonon coupling in a single $\mathrm{Au}$ film irradiated by a femtosecond pulse laser. They showed that electron-phonon coupling behavior depends significantly on the laser fluence and should be carefully determined when describing non-equilibrium energy transport. For the present study, we used the theoretical model proposed by Lin et al. ${ }^{13)}$ to ensure consideration of electron scattering away from the Fermi surface at high electron temperatures. The rigorous form of temperature-dependent electron-phonon coupling can be expressed as: ${ }^{13)}$

$$
G\left(T_{\mathrm{e}}\right)=\frac{\pi \hbar k_{\mathrm{B}} \lambda\left\langle\omega^{2}\right\rangle}{g\left(\varepsilon_{\mathrm{F}}\right)} \int_{-\infty}^{\infty} g^{2}(\varepsilon)\left(-\frac{\partial f}{\partial \varepsilon}\right) d \varepsilon,
$$

where $g(\varepsilon)$ is the electron DOS at the energy level $\varepsilon$. The above equation reflects a major variation in the $G$-factor at high electron temperatures, whereas it requires some difficult procedures for electronic structure calculation of the electron DOS.

Similar to the previous work of Ashcroft and Mermin, ${ }^{26)}$ we used the conventional heat conduction model in a $\mathrm{SiO}_{2}$ layer to calculate the lattice phonon temperature on a dielectric side in the present study. This approach is valid under our conditions of laser irradiation in which electrons in the valance band of $\mathrm{SiO}_{2}$ are not excited beyond a band gap energy of $9.0 \mathrm{eV}$ for $\mathrm{SiO}_{2} \cdot{ }^{18,26-28)}$ Therefore, the energy transport via electrons can be neglected in $\mathrm{a} \mathrm{SiO}_{2}$ layer where conductive heat transfer becomes dominant. We used the simple conduction equation for a dielectric $\mathrm{SiO}_{2}$ layer: 


$$
\rho_{\mathrm{sm}} \frac{\partial C_{\mathrm{sm}} T}{\partial t}=\frac{\partial}{\partial z}\left(k_{\mathrm{sm}} \frac{\partial T}{\partial z}\right)
$$

where $\rho_{\mathrm{sm}}, C_{\mathrm{sm}}$, and $k_{\mathrm{sm}}$ are the density, heat capacity, and thermal conductivity of $\mathrm{SiO}_{2}$, respectively.

\subsection{Thermal boundary resistance (TBR) and numerical details}

Many researchers have used the acoustic mismatch model (AMM) or the diffuse mismatch model (DMM) for TBR at a solid-solid interface. ${ }^{29-31)}$ In these previous studies, AMM and DMM considering only the phonon-phonon resistance were used for energy transfer at the metalnonmetal interface under the assumption that electronphonon coupling resistance can be ignored. However, Majumdar and Reddy ${ }^{20)}$ suggested electron-phonon coupling resistance at the interface by assuming that in the metal, $k_{\mathrm{e}} \gg k_{\mathrm{p}}$ such that the harmonic mean of the electronic and lattice thermal conductivities is equal to $k_{\mathrm{p}}$. The simplified relation of the interfacial conductance can be written as

$$
h \approx \frac{\sqrt{G k_{\mathrm{p}}}}{\left[1+\frac{\sqrt{G k_{\mathrm{p}}}}{h_{\mathrm{pp}}}\right]}=\frac{h_{\mathrm{ep}} h_{\mathrm{pp}}}{h_{\mathrm{ep}}+h_{\mathrm{pp}}},
$$

where $h_{\text {ep }}$ can be expressed as $\left(G k_{\mathrm{p}}\right)^{1 / 2}$, and is the electronphonon thermal conductance in metal. ${ }^{20)}$ Additionally, $k_{\mathrm{p}}$ denotes the thermal conductivity in metal and $h_{\mathrm{pp}}$ indicates the phonon-phonon thermal conductance per unit area that can be predicted using DMM. According to Majumdar and Reddy, ${ }^{20}$ at sufficiently low temperatures, $h_{\mathrm{pp}}$ can be expressed by $\xi T^{3}$, where $T$ denotes the lattice temperature on the dielectric side and $\xi$ is the empirical constant. In the present calculation, the empirical constant $\xi$ is assumed to be unity at phonon temperatures less than the melting temperature of $\mathrm{SiO}_{2}$. At a higher phonon temperature, the role of electron-phonon coupling becomes more important, and even dominant in certain high temperature regimes. ${ }^{20)}$ As shown in eq. (11), the TBR is closely associated with the electronphonon coupling factor, which is substantially dependent on the electron energy excited by the femtosecond pulse laser. Equation (11) includes two resistances: electron-phonon coupling resistance $\left(R_{\mathrm{ep}}=1 / h_{\mathrm{ep}}\right)$ and phonon-phonon resistance at the interface $\left(R_{\mathrm{pp}}=1 / h_{\mathrm{pp}}\right)$. The interfacial thermal conductivity $k_{\text {in }}$ can be determined by $h L$, where $L$ is the characteristic length.

For discretization of the governing equations (see eqs. (1) and (2)) in the present study, the finite difference method (FDM) was used with the fully implicit scheme. For the diffusion terms, a central differencing scheme with second order accuracy was used, and integration of the transient terms was performed over the control volume and time interval from $t$ to $t+\Delta t$. The algebraic discretized equations were solved by the strongly implicit procedure (SIP). ${ }^{32,33)}$ Figure 1 shows schematics of the $\mathrm{Au} / \mathrm{SiO}_{2}$ multilayer structure irradiated by femtosecond pulse lasers. $d_{\mathrm{f}}, d_{\mathrm{s}}$, and $d_{\text {in }}$ denote the depths of the $\mathrm{Au}$ film, $\mathrm{SiO}_{2}$ substrate, and interface, respectively. Additionally, $T_{\text {int,top }}$ and $T_{\text {int,bottom }}$ are the temperatures at the top and bottom surfaces of the interface region, respectively.

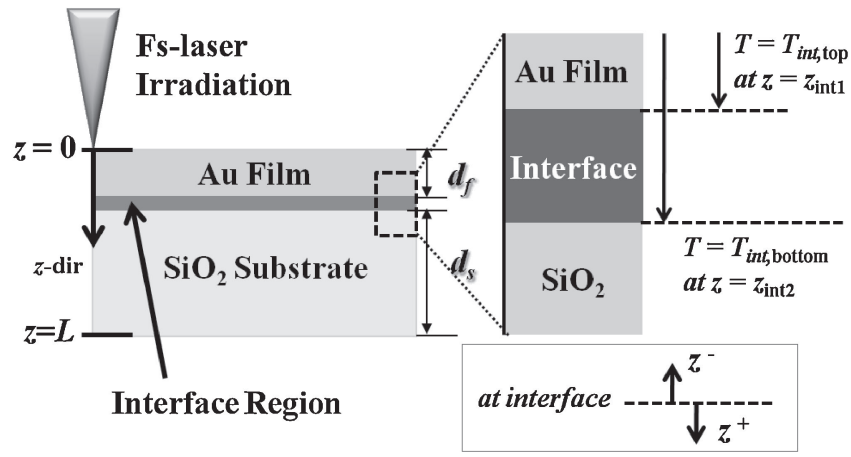

Fig. 1 Schematics of the $\mathrm{Au} / \mathrm{SiO}_{2}$ film structure and the corresponding interface region between the two dissimilar films.

The von Neumann boundary conditions with zero gradient at $z=0$ and $z=L$ were used for the temperatures of the electrons and lattice phonons under the assumption that during a short period of laser heating, heat loss at the top and bottom surfaces is neglected. ${ }^{34)}$ The boundary conditions are as follows:

$$
\begin{aligned}
& \left.\frac{\partial T_{\mathrm{e}}}{\partial z}\right|_{z=0}=\left.\frac{\partial T_{1}}{\partial z}\right|_{z=0}=0, \\
& \left.\frac{\partial T_{\mathrm{e}}}{\partial z}\right|_{z=L}=\left.\frac{\partial T_{1}}{\partial z}\right|_{z=L}=0 .
\end{aligned}
$$

In addition, the electron and phonon temperatures were initially set at $300 \mathrm{~K}$, indicating that the electrons and phonons are initially in a thermal equilibrium state. As shown in Fig. 1, there is an interface layer with a finite depth between two dissimilar films. The depth of the interface layer is very difficult to measure accurately because of nonuniformity and a very small scale (on the order of a few nanometers). An interface thickness of one nanometer was thus assumed in the present study, and only one computational node was located at the center of the interface region to numerically couple the metal and dielectric sides. A nanometer interface thickness was determined from the preliminary tests. As shown in Fig. 1, the interfacial boundary condition of the electron temperature at $z=z_{\text {int1 }}$, can be written as

$$
\left.k_{\mathrm{e}} \frac{d T_{\mathrm{e}}}{d z}\right|_{z=z_{\text {int } 1}}=0 .
$$

The insulation condition for electrons in the metal side was used in the present study because of the absence of electrons in the dielectric layer. This condition is valid when the transferred electron energy does not exceed $9.0 \mathrm{eV}$, which is the band gap energy of the $\mathrm{SiO}_{2}$ layer, indicating that the phonon energy transport becomes dominant at the interface between the metal and dielectric layers. Meanwhile, for the lattice phonons, the continuity of the phonon energy flux at both sides of the interface gives

$$
\begin{aligned}
& \left.k_{\mathrm{p}} \frac{d T_{\mathrm{p}}}{d z}\right|_{z=z_{\text {int1 }}^{-}}=\left.k_{\text {in }} \frac{d T_{\mathrm{p}}}{d z}\right|_{z=z_{\text {int1 }}^{+}} \\
& \left.k_{\text {in }} \frac{d T_{\mathrm{p}}}{d z}\right|_{z=z_{\text {int2 }}^{-}}=\left.k_{\mathrm{p}} \frac{d T_{\mathrm{p}}}{d z}\right|_{z=z_{\text {int2 }}^{+}},
\end{aligned}
$$


Table 1 Thermal and optical properties of the Au film and $\mathrm{SiO}_{2}$.

\begin{tabular}{|c|c|c|c|}
\hline & Description & Value & Reference \\
\hline \multirow{6}{*}{$\mathrm{Au}$} & Electron number density, $n_{\mathrm{e}}$ & $5.9 \times 10^{28}\left[\mathrm{~m}^{-3}\right]$ & Ref. 9, 26) \\
\hline & Mass of ion, $M$ & 196.97 [a.m.u] & Ref. 23) \\
\hline & Debye temperature, $T_{\mathrm{D}}$ & $170[\mathrm{~K}]$ & \multirow{4}{*}{ Ref. 26) } \\
\hline & Melting temperature, $T_{\text {melt }}$ & $1337[\mathrm{~K}]$ & \\
\hline & Fermi temperature, $T_{\mathrm{F}}$ & $6.39 \times 10^{4}[\mathrm{~K}]$ & \\
\hline & First ionization potential, $J_{\mathrm{i}}$ & $9.22[\mathrm{eV}]$ & \\
\hline \multirow{3}{*}{$\mathrm{SiO}_{2}$} & Density, $\rho_{\mathrm{sm}}$ & $2.2\left[\mathrm{~g} / \mathrm{cm}^{3}\right]$ & \multirow{3}{*}{ Ref. 27, 28) } \\
\hline & Heat capacity constant, $C_{\mathrm{sm}}$ & $740[\mathrm{~J} / \mathrm{kg} \cdot \mathrm{K}]$ & \\
\hline & Thermal conductivity, $k_{\mathrm{sm}}$ & $1.38[\mathrm{~W} / \mathrm{m} \cdot \mathrm{K}]$ & \\
\hline
\end{tabular}

where the positive and negative signs in the superscripts denote the downward and upward directions at the interface, as illustrated in Fig. 1. With an initial constant electron number density of $5.9 \times 10^{28} \mathrm{~m}^{-39,26)}$ in the $\mathrm{Au}$ film, the numerical simulations were conducted using eqs. (1), (2), and (10) and the initial and boundary conditions mentioned above. The converged solutions could be obtained when the residuals of the discretized equations were less than $10^{-6}$. The physical properties of the Au film used in the present study and $\mathrm{SiO}_{2}$ are listed in Table 1. In this study, numerical simulations were first conducted in order to evaluate the computational code for a $200-\mathrm{nm}$ single $\mathrm{Au}$ film irradiated by a 100-fs pulse laser with a 1053-nm wavelength. After the code validation, we conducted extensive simulations for a metal-dielectric film structure that consisted of a bulk $\mathrm{SiO}_{2}$ substrate with a $0.6 \mu \mathrm{m}$ depth and a $100-\mathrm{nm} \mathrm{Au}$ film deposited onto the substrate. For all calculations, a 100-fs pulse laser with a 1053-nm wavelength was used, whereas a very low laser fluence of $0.001 \mathrm{~J} / \mathrm{cm}^{2}$ for a single film calculation was used for comparison with the experimental data. Laser fluence was varied from 0.01 to $0.3 \mathrm{~J} / \mathrm{cm}^{2}$ for calculations for the $\mathrm{Au}-$ $\mathrm{SiO}_{2}$ film structure.

\section{Results and Discussion}

Prior to the main discussion, we attempt to examine the thermal responses of the physical properties depending on energy carriers because thermal properties and energy carriers are highly associated with the TBR during laser irradiation. Figure 2 shows the spatial distribution of the electron thermal conductivity that is closely related to the electron temperature. These results were obtained from the preliminary simulation for a $300-\mathrm{nm}$ single Au film irradiated by a 100 -fs pulse laser at a fluence of $0.1 \mathrm{~J} / \mathrm{cm}^{2}$ with a 1053 $\mathrm{nm}$ wavelength. The electron thermal conductivity increased at the top surface with the increase in electron heat capacity. ${ }^{9,24)}$ The electron thermal conductivity did not change at the bottom surface of the Au film due to the fact that the electron temperature was lower than the melting temperature. If the electron temperature is higher than $1337 \mathrm{~K}$, the electron collision frequency lies in the hot plasma regime where electron conductivity gradually increases. The variation in electron thermal conductivity can directly affect energy transfer in gold film structures. ${ }^{24)}$ For

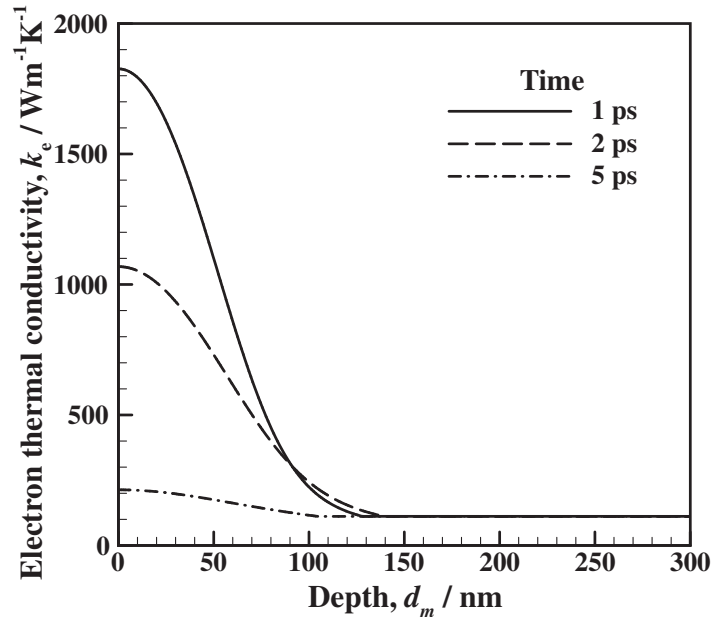

(a)

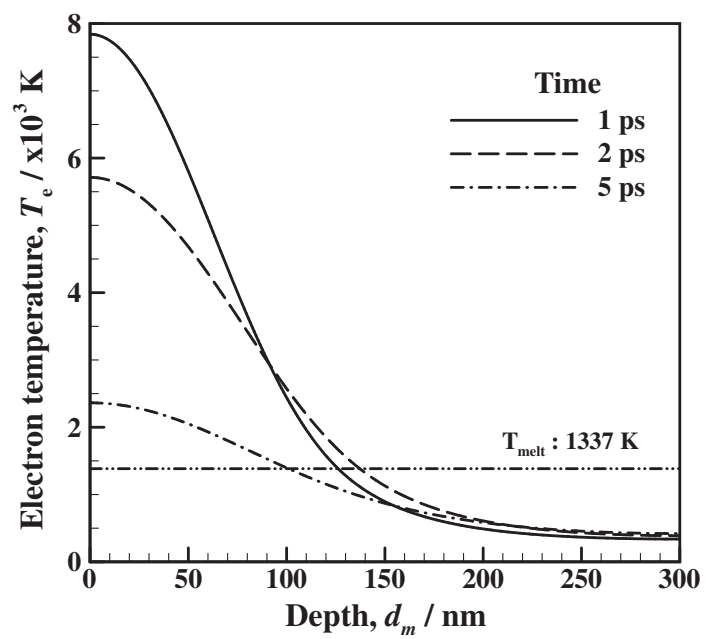

(b)

Fig. 2 The spatial variation of the electron thermal conductivity of a 300$\mathrm{nm}$ single Au layer exposed to a 100 -fs pulse laser with $0.1 \mathrm{~J} / \mathrm{cm}^{2}$ irradiation.

validating our computational code, we conducted numerical simulations for a 200-nm Au film irradiated by a femtosecond pulse laser with $F=0.001 \mathrm{~J} / \mathrm{cm}^{2}$ and $t_{\mathrm{P}}=100 \mathrm{fs}$. The predicted electron temperatures were compared with the numerical results reported previously by Sim and Lee ${ }^{24)}$ and experimental results. ${ }^{35)}$ As shown by the transient evolution of electron temperatures in Fig. 3, the change in normalized electron temperature was defined as $\left(T_{\mathrm{e}}-T_{\mathrm{eq}}\right) /$ $\left(T_{\mathrm{e}}-T_{\mathrm{eq}}\right)_{\max }$, which is analogous to the normalized reflectivity change and similar to literature. ${ }^{12)}$ As a result, our numerical predictions show good agreement with the experimental data and previous results. ${ }^{24)}$

Even when previous researchers assumed that the interface layer was very thin, ${ }^{18)}$ they were unable to suggest a suitable thickness and their estimations were on the order of a few nanometers. ${ }^{18,29,36)}$ In the present study we created an interfacial region between the $\mathrm{Au}$ film and $\mathrm{SiO}_{2}$ substrate for convenient modeling of a metal-nonmetal interface region, as shown in Fig. 1. Unfortunately, it is impossible to accurately determine a finite thickness of this interface region. Figure 4 shows the preliminary results of numerical 


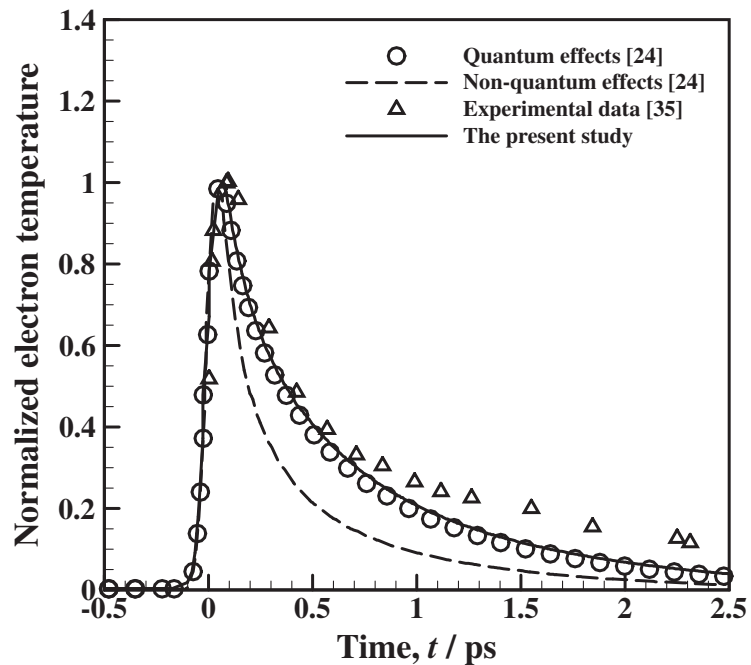

Fig. 3 Comparison of the electron temperatures among the numerical results from Sim and Lee, ${ }^{24)}$ experimental data from Bronson et al. ${ }^{35)}$ and the present predictions at the top surface of a 200-nm single Au film after $0.001 \mathrm{~J} / \mathrm{cm}^{2}$ irradiation.

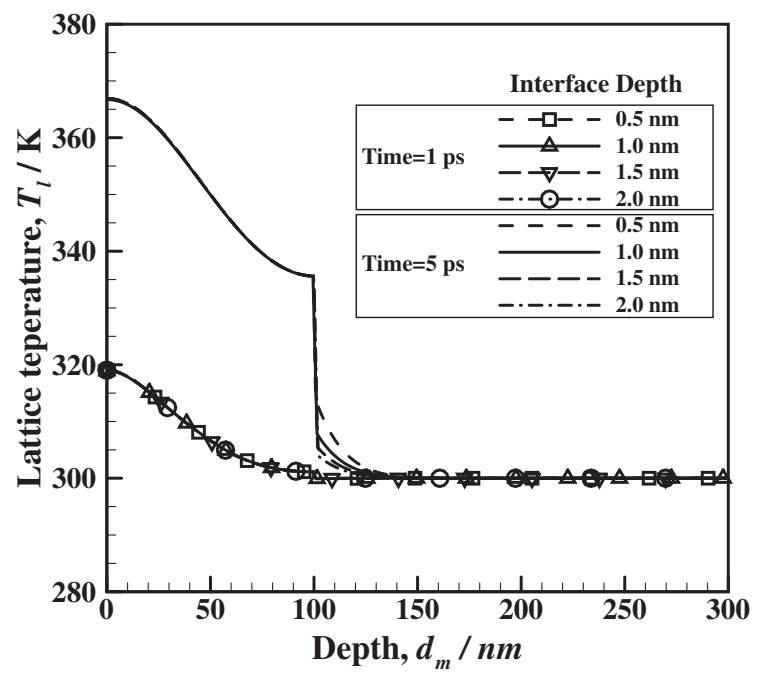

Fig. 4 Comparison of the variation of lattice temperature according to different interface depths $(0.5,1.0,1.5$, and $2.0 \mathrm{~nm})$ at $1 \mathrm{ps}$ and $5 \mathrm{ps}$ after irradiation on $\mathrm{Au} / \mathrm{SiO}_{2}$ multilayer film (at $F=0.01 \mathrm{~J} / \mathrm{cm}^{2}$ and $\left.t_{\mathrm{p}}=100 \mathrm{fs}\right)$.

sensitivity for various thicknesses of the interface region. The lattice phonon temperature distributions are shown at 1.0 and $5.0 \mathrm{ps}$ after fs-laser irradiation was completed. At $t=1.0 \mathrm{ps,}$ a substantial temperature difference was observed at the interface, indicating the existence of TBR. Alternatively, the TBR effect was drastically reduced at $t=5.0 \mathrm{ps}$. In particular, the differences among the lattice temperatures estimated for different thicknesses of the interface region were very small, and the maximum difference was less than approximately $5.0 \mathrm{~K}$, suggesting that the numerical dependency on the thickness of the interface region can be sufficiently neglected. Therefore, the thickness of the interface region was set at one nanometer for all calculations.

Figure 5 predicts the change in electron temperature in the depth direction at different times. The electron temper-

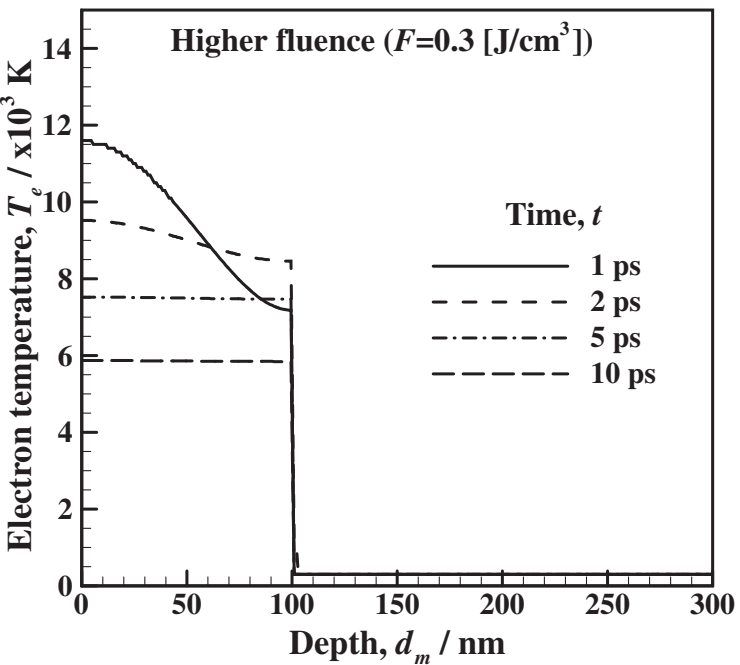

Fig. 5 The change of electron temperature in the depth direction at different times $(1,2,5$, and $10 \mathrm{ps})$.

ature at the top surface rapidly increased during laser irradiation. After the increase, the temperature slowly decreased because of electron-phonon energy transport and electron-electron thermal conduction. The electron temperature at the interface gradually increased due to electronelectron thermal conduction. For electrons in dielectric materials (electrical insulators) to be excited, they must have absorbed energy greater than the $9.0 \mathrm{eV}$ band gap energy of $\mathrm{SiO}_{2},{ }^{27,28)}$ above which the electrons in the valence bands can move toward the conduction band. However, under the present conditions of laser irradiation, the electrons in the valance band of $\mathrm{SiO}_{2}$ were not excited beyond $9.0 \mathrm{eV}$. For instance, at $F=0.3 \mathrm{~J} / \mathrm{cm}^{2}$, the maximum electron temperature at the interface was estimated to be $1.16 \mathrm{eV}$, which was much less than $9.0 \mathrm{eV}$.

Figure 6 compares the predicted spatial distributions of lattice temperatures for low and high laser fluences. The lattice temperature rapidly decreased at the interface due to the TBR, which is affected by the lattice temperature and electron-phonon coupling. Indeed, the TBR affects the rapid decrease in interfacial thermal conductivity. As shown in Fig. 6(a), a temperature difference between the top and bottom surfaces of the interface region for low laser fluence $\left(F=0.01 \mathrm{~J} / \mathrm{cm}^{2}\right)$ was maintained up to approximately $58 \%$ of the maximum lattice temperature estimated at $t=5.0 \mathrm{ps}$. Alternatively, at higher laser fluence $\left(F=0.3 \mathrm{~J} / \mathrm{cm}^{2}\right)$, the TBR effect can be neglected after $t=5 \mathrm{ps,} \mathrm{as} \mathrm{shown} \mathrm{in}$ Fig. 6(b). Based on these results, we can conclude that the TBR at the interface was dominant at the early stage of laser irradiation for a very short time, and then drastically decreased with time. Hence, the interfacial thermal conductivity increased with time and its magnitude was nearly the same as the thermal conductivity of $\mathrm{SiO}_{2}$.

Majumdar and Reddy ${ }^{20}$ calculated the thermal conductance using a constant value of the electron-phonon coupling factor (so-called, $G$-factor), and reported that the existing TBRs were affected only by lattice temperature. Unlike the previous work, ${ }^{20)}$ we attempted to estimate TBRs in the present study by considering a non-linear change in the 


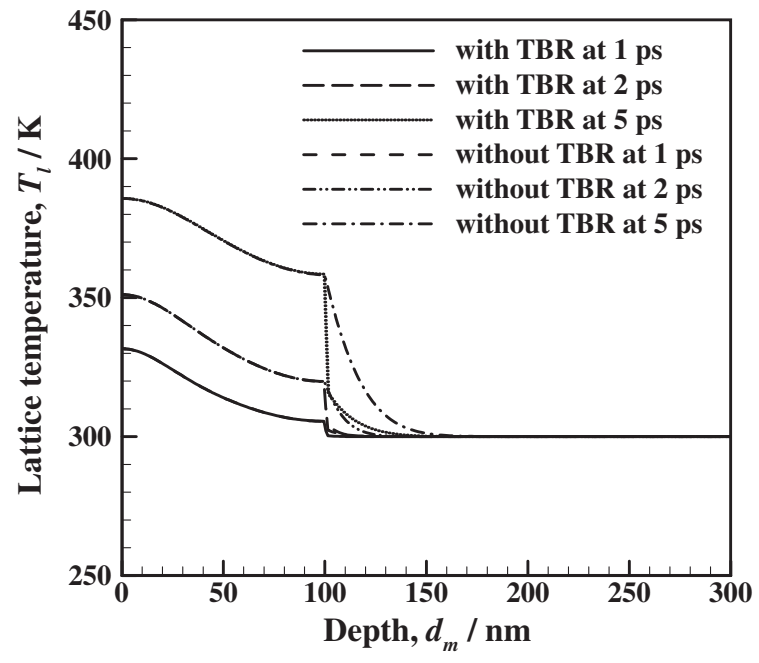

(a) $F=0.01 \mathrm{~J} / \mathrm{cm}^{2}$

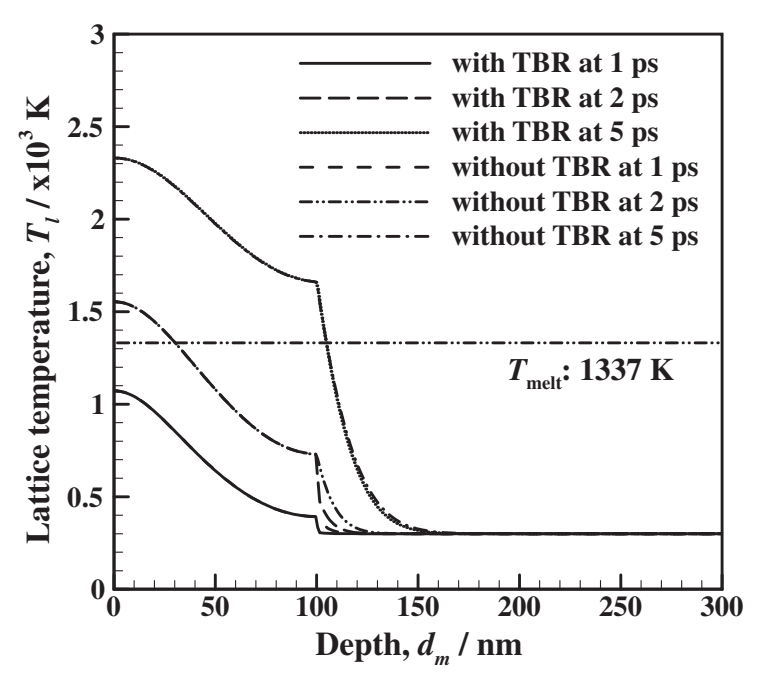

(b) $F=0.3 \mathrm{~J} / \mathrm{cm}^{2}$

Fig. 6 Comparison of the change of the lattice temperature with respect to depth when the $\mathrm{Au} / \mathrm{SiO}_{2}$ multilayer irradiated by a femtosecond laser with low $\left(0.01 \mathrm{~J} / \mathrm{cm}^{2}\right)$ and high laser fluences $\left(0.3 \mathrm{~J} / \mathrm{cm}^{2}\right)$ with and without TBR effects.

$G$-factor during laser irradiation. This is a clear difference between our study and the previous work of Majumdar and Reddy ${ }^{20)}$ and it is very important as the interfacial thermal conductance is closely associated with the electron-phonon coupling. In addition, our results may be helpful in understanding the major role of $\mathrm{TBR}$ at the interfaces during a very short time period in which very high photon energy absorption occurs. Figure 7 illustrates the transient evolution of TBR, the lattice temperature of the interface region, $G$-factor, and electron temperature. For this case, a $100-\mathrm{nm} \mathrm{Au}$ film and a relatively higher laser fluence of $0.3 \mathrm{~J} / \mathrm{cm}^{2}$ were used for the simulation. In Fig. 7(a) it is apparent that as the laser fluence increased, the rate at which TBR was decreasing rapidly increased due to increased electron-phonon coupling. Hence, at higher laser fluence, the lattice temperature increased faster than when the laser fluence was lower. Eventually, the TBR reached a saturated value, which depended on the lattice temperature. It is evident in Fig. 7(b) that the $G$-factor rapidly increased with

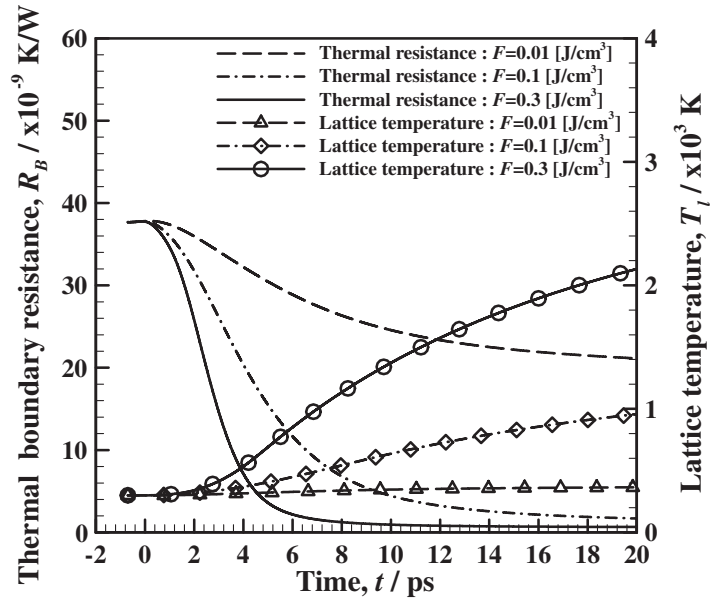

(a)

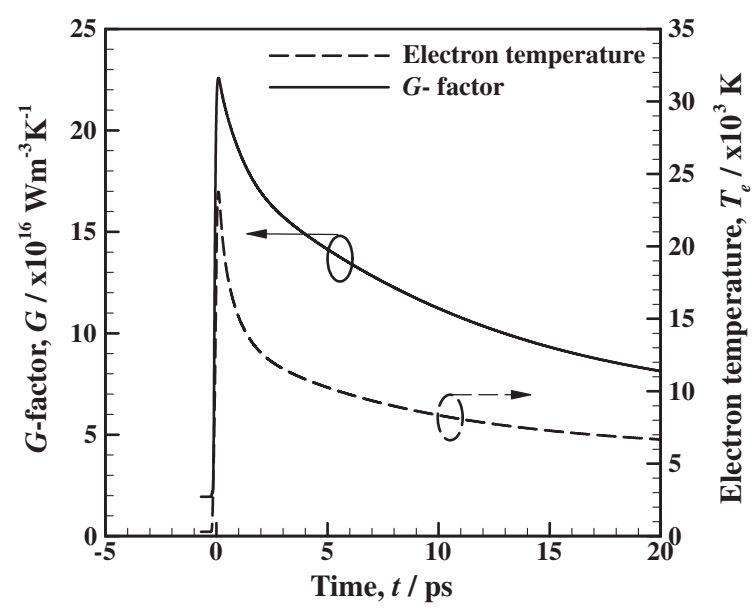

(b)

Fig. 7 (a) The prediction of the variation of thermal resistance and lattice temperature with respect to time, and (b) transient behavior of electron temperature and electron-phonon coupling factor $\left(F=0.3 \mathrm{~J} / \mathrm{cm}^{2}\right.$, $\left.t_{\mathrm{P}}=100 \mathrm{fs}, d_{\mathrm{f}}=100 \mathrm{~nm}\right)$.

the electron temperature of the Au film during laser irradiation. This result supports the fact that TBR is strongly dependent on the behavior of electron-phonon coupling and electron temperature.

Figure 8 shows the transient evolution of the temperature difference estimated at the top and bottom surfaces of the interface region. A large difference (approximately $150 \mathrm{~K}$ ) exists between the results obtained with and without considering the TBR effect, indicating that the presence of TBR should be considered for more accurate prediction of the drop in lattice temperature occurring at the interface. At the early stage of laser irradiation, the temperature drop substantially increased at the interface region, and gradually decreased with time. Additionally, the maximum temperature difference is achieved more quickly with increasing laser fluence due to variation in the rates of lattice temperature increase in the $\mathrm{Au}$ film. Moreover, the maximum temperature difference of the interface region reached $32 \%$ of the maximum lattice temperature estimated at $t=2.0 \mathrm{ps}$ for $F=0.3 \mathrm{~J} / \mathrm{cm}^{2}$, indicating that the temperature drop at the interface region may cause thermal damage in a multilayered structure. ${ }^{14)}$ 


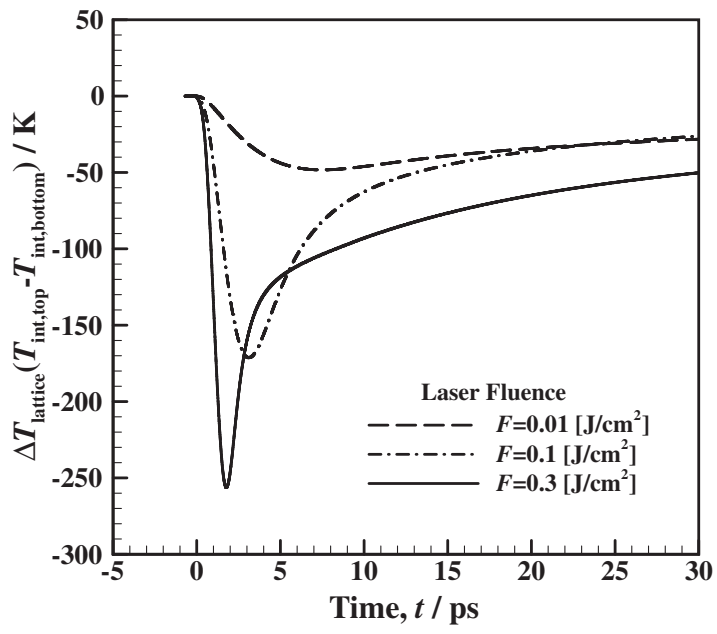

(a) with TBR

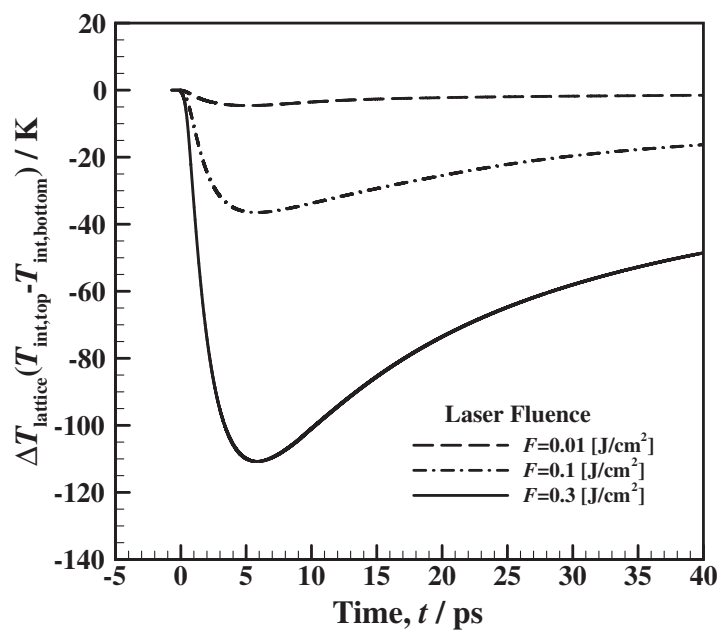

(b) without TBR

Fig. 8 The comparison of the magnitude of temperature difference with time at various laser fluences $\left(0.01,0.1,0.3 \mathrm{~J} / \mathrm{cm}^{2}\right)$ : (a) with and (b) without TBR effects.

\section{Conclusions}

In the present study we numerically investigated TBR effects on non-equilibrium energy transport in a multilayer $\mathrm{Au} / \mathrm{SiO}_{2}$ film structure irradiated by a femtosecond pulse laser. We established a combined set of energy equations to describe electron-phonon and phonon-phonon interactions and applied the thermal conductance model to $\mathrm{Au} / \mathrm{SiO}_{2}$ films heated by ultra-short pulse laser interactions. Based on our findings, the following conclusions can be drawn:

(1) The TBR rapidly increased thermal conductivity at the interface. The temperature difference at the interface was maintained up to approximately $58 \%$ of the maximum lattice temperature at $t=5.0 \mathrm{ps}$ for $F=$ $0.01 \mathrm{~J} / \mathrm{cm}^{2}$, whereas the TBR did not affect the temperature difference after $t=5.0 \mathrm{ps}$ for high laser fluence. The TBR at the interface was dominant at an early stage of laser irradiation for a very short time, and it drastically decreased with time.

(2) The rate at which TBR was decreasing significantly increased due to the increased electron-phonon cou- pling with the increase in laser fluence. At higher laser fluence, the lattice temperature increased faster than when the laser fluence was lower. Eventually, the TBR reached a saturated value, which depended on the lattice temperature.

(3) A large difference in lattice temperature at the interface was observed and the lattice temperature difference reached $32 \%$ of the maximum lattice temperature estimated at $t=2.0 \mathrm{ps}$ for $F=0.3 \mathrm{~J} / \mathrm{cm}^{2}$, indicating that the temperature drop in the interface region may cause thermal damage in a multilayered structure. The maximum temperature difference at the interface was reached more rapidly as the laser fluence increased due to variation in the rates of lattice temperature increase in the Au films.

(4) In spite of a rapid increase in the lattice temperature of the $\mathrm{Au}$ film, the phonon energy was rarely transferred from the $\mathrm{Au}$ film to the $\mathrm{SiO}_{2}$ substrate due to the existence of TBR. Additionally, TBR was strongly dependent on the behavior of electron-phonon coupling and electron temperature. Therefore, the presence of TBR should be considered to ensure more accurate prediction of the lattice temperature drop occurring at the interface.

\section{Acknowledgements}

This work was sponsored by the Human Resources Development of the Korea Institute of Energy Technology Evaluation and Planning (KETEP) grant funded by the Korea government, Ministry of Knowledge and Economy (2008-PEP-HM-E-05-0000).

\section{REFERENCES}

1) P. F. Sharp, A. Manivannan, H. Xu and J. V. Forrester: Phys. Med. Biol. 49 (2004) 1085-1086.

2) I. M. Burakov, N. M. Bulgakova, R. Stoian, A. Rosenfuld and I. V. Hertel: Appl. Phys. A 81 (2005) 1639-1645.

3) K. Claytor, S. Khatua, J. M. Guerrero, A. Tcherniak, J. M. Tour and S. Link: J. Chem. Phys. 130 (2009) 164710-164719.

4) X. Zhang, D. D. Zhang, H. Liu, H. F. Xu, M. X. Jin and D. J. Ding: J. Phys. B 43 (2010) 025102-025108.

5) H. S. Sim, K. G. Kang, S. H. Lee, J. M. Kim and Y. E. Shin: Mater. Sci. Forum 580-582 (2008) 143-146.

6) K. G. Kang, S. H. Lee, H. S. Ryou, Y. K. Choi, S. H. Park and J. S. Lee: Mater. Trans. 49 (2008) 1880-1888.

7) S. I. Anisimov, B. L. Kapeliovich and T. L. Terel'man: Sov. Phys. JETP 39 (1974) 375-377.

8) J. K. Chen and J. E. Beraun: J. Opt. A: Pure and Appl. Opt. 5 (2003) $168-173$.

9) K. Eidmann, J. Meyer-ter-Vehn, T. Schlegel and S. Huller: Phys. Rev. E 62 (2000) 1202-1214.

10) K. Furusawa, K. Takahashi, H. Kumagai, K. Midorikawa and M. Obara: Appl. Phys. A 69 (1999) S359-366.

11) L. Jiang and H. L. Tsai: Proc. ASME Heat Transfer Summer Conference, (San Francisco, CA, USA, 2005) No. HT2005-72199.

12) T. Q. Qui and C. L. Tien: ASME J. Heat Transfer 115 (1993) 835-841.

13) Z. Lin, L. V. Zhigilei and V. Celli: Phys. Rev. B 77 (2008) 075133.

14) K. Ramadan and M. A. Al-Nimr: Int. J. Thermal Sci. 48 (2009) 17181727.

15) W. M. G. Ibrahim, H. E. Elsayed-Ali, C. E. Bonner and M. Shinn: Int. J. Heat Mass Transfer 47 (2004) 2261-2268.

16) A. M. Chen, H. F. Xu, Y. F. Jiang, L. Z. Sui, D. J. Ding, H. Liu and M. X. Jin: Appl. Surf. Sci. 257 (2010) 1678-1683. 
17) G. Chen: J. Heat Trans. 119 (1997) 220-229.

18) H. C. Chien, D. J. Yao and C. T. Hsu: Appl. Phys. Lett. 93 (2008) 231910.

19) P. E. Hopkins, J. L. Kassebaum and P. M. Norris: J. Appl. Phys. 105 (2009) 023710

20) A. Majumdar and P. Reddy: Appl. Phys. Lett. 84 (2004) 23.

21) E. S. Landry and A. J. McGaughey: Phys. Rev. B 80 (2009) 165304.

22) C. L. Tien, A. Majumdar and F. M. Gerner: Microscale Energy Transport, (Taylor \& Francis 1998).

23) C. Kittel: Introduction to Solid State Physics, (John Wiley \& Sons 1986).

24) H. S. Sim and S. H. Lee: Numerical Heat Transfer Part A 54 (2008) 279-292.

25) J. B. Lee, K. G. Kang and S. H. Lee: Mater. Trans. 52 (2011) 547-553.

26) N. W. Ashcroft and N. D. Mermin: Solid State Physics, (Thomson Learning, 1976).

27) M. J. Weber: Handbook of optical materials, (CRC Press Laser and Optical Science and Technology Series 2003).

28) G. Neudeck: The PN Junction Diode, (Addison-Wesley Publishing Co. 1989).

29) D. G. Cahill, W. K. Ford, K. E. Goodsoon, G. D. Mahan, A. Majumdar, H. J. Maris, R. Merlin and S. R. Phillpot: J. Appl. Phys. 93 (2003) 793 818.

30) Y. S. Ju, M. T. Hung and T. Usui: J. Heat Transfer 128 (2006) 919-925

31) E. T. Swartz and R. O. Pohl: Rev. Mod. Phys. 61 (1989) 605-668.

32) S. V. Patankar: Numerical heat transfer and fluid flow, (Taylor \& Fransis, 1980)

33) H. K. Versteeg and W. Malalasekera: An introduction to computational fluid dynamics, (Longman, 1995).

34) S. H. Lee, H. S. Sim, J. Lee, J. M. Kim and Y. E. Shin: Mater. Trans. 47 (2006) 2835-2841.

35) S. D. Bronson, A. Kazeroonian, J. S. Moodera, D. W. Face, T. K. Cheng, E. P. Ippen, M. S. Dresselhaus and G. Dresselhaus: Phys. Rev. Lett. 64 (1990) 2173-2175.

36) W. L. Chan, R. S. Averback and D. G. Cahill: Appl. Phys. A 97 (2009) 287-294.

\section{Nomenclature}

$C_{\text {e }}$ electron heat capacity, $\mathrm{W} / \mathrm{m}^{3}$

$C_{1}$ lattice heat capacity, $\mathrm{W} / \mathrm{m}^{3}$

$C_{\text {sm }}$ heat capacity of $\mathrm{SiO}_{2}, \mathrm{~W} / \mathrm{m}^{3}$

$d_{\mathrm{f}}$ film thickness, $\mathrm{m}$

$d_{\text {in }}$ interface depth, $\mathrm{m}$

$d_{\mathrm{m}}$ multilayered structure thickness, $\mathrm{nm}$

$d_{\mathrm{s}}$ substrate thickness, $\mathrm{m}$

$E_{\mathrm{g}}$ band gap energy, $\mathrm{J}$

$G$ electron-phonon coupling constant, $\mathrm{W} / \mathrm{m}^{3} \mathrm{~K}$

$g(\varepsilon)$ electron density of states (DOS), states $/ \mathrm{eV} /$ atom

$\hbar$ reduced Planck constant, Js

$h$ thermal conductance, $\mathrm{W} / \mathrm{m}^{2} \mathrm{~K}$

$I$ laser intensity, $\mathrm{W} / \mathrm{m}^{2}$

$F$ laser fluence, $\mathrm{J} / \mathrm{cm}^{2}$

$J_{\mathrm{i}}$ ionization potential, $\mathrm{eV}$

$k$ extinction coefficient

$k_{\mathrm{e}}$ electron thermal conductivity, $\mathrm{W} / \mathrm{mK}$

$k_{1}$ lattice thermal conductivity, $\mathrm{W} / \mathrm{mK}$

$k_{\mathrm{B}}$ Boltzmann constant, Js

$k_{\mathrm{s}}$ empirical constant

$k_{\text {sm }}$ thermal conductivity of $\mathrm{SiO}_{2}, \mathrm{~W} / \mathrm{mK}$

$L$ characteristic length, $\mathrm{m}$
$M$ ion mass, a.m.u

$m_{\mathrm{e}}$ electron mass, $\mathrm{kg}$

$n_{\mathrm{i}}$ ion number density, $/ \mathrm{m}^{3}$

$n_{\mathrm{e}}$ electron number density, $/ \mathrm{m}^{3}$

$n$ refractive index

$r_{\mathrm{o}}$ ion sphere radius, $\mathrm{m}$

$R$ reflectivity

$R_{\mathrm{B}}$ thermal boundary resistance, $\mathrm{K} / \mathrm{W}$

$S$ laser heating source term, $\mathrm{W} / \mathrm{m}^{3}$

$T$ lattice temperature on the nonmetal side, $\mathrm{K}$

$T_{\mathrm{e}}$ electron temperature, $\mathrm{K}$

$T_{1}$ phonon (lattice) temperature on the metal side, $\mathrm{K}$

$T_{\mathrm{D}}$ Debye temperature, $\mathrm{K}$

$T_{\mathrm{F}}$ Fermi temperature, $\mathrm{K}$

$T_{\text {int,top }}$ temperature at the top surface of interface regime, $\mathrm{K}$

$T_{\text {int,bottom }}$ temperature at the bottom surface of interface regime, $\mathrm{K}$

$T_{\text {melt }}$ melting temperature, $\mathrm{K}$

$t$ time, $\mathrm{s}$

$t_{\mathrm{p}}$ pulse duration time (FWHM), s

$V_{\mathrm{e}}$ electron velocity, $\mathrm{m} / \mathrm{s}$

$z$-direction, $\mathrm{m}$

$Z$ ionization degree

\section{Greek symbols}

$\alpha$ linear absorption coefficient, /m

$\beta$ two-photon absorption coefficient, $\mathrm{m} / \mathrm{W}$

$\varepsilon$ average kinetic energy of electron, $\mathrm{eV}$

$\varepsilon_{1}$ real part of dielectric function

$\varepsilon_{2}$ imaginary part of dielectric function

$\varepsilon$ dielectric function

$\lambda$ laser wavelength, $\mathrm{m}$

$\lambda_{\mathrm{e}}$ electron mean free path, $\mathrm{m}$

$\mu$ the chemical potential, $\mathbf{J}$

$\tau_{\mathrm{e}}$ electron-electron relaxation time, $\mathrm{s}$

ln $\Lambda$ Coulomb logarithm

$v$ collision frequency, $/ \mathrm{s}$

$\rho_{\text {sm }}$ density of $\mathrm{SiO}_{2}, / \mathrm{m}^{3}$

$\omega_{\mathrm{L}}$ laser frequency, $/ \mathrm{s}$

$\omega_{\mathrm{p}}$ plasma frequency, /s

$\hbar$ reduced Planck constant, Js

$\hbar \omega$ photon energy quantum

Superscripts

0 old values at time $t$

Subscripts

CV control volume

e electron

ep electron-phonon

$\mathrm{e}^{*}$ interface between nodes

f films

i ion

1 lattice

int interface

pp phonon-phonon

s substrate 